\title{
AN INFINITE-DIMENSIONAL CLASSICAL INTEGRABLE SYSTEM AND THE HEISENBERG AND SCHRÖDINGER REPRESENTATIONS
}

\author{
Anthony M. BLOCH \\ Department of Mathematics, The University of Michigan, Ann Arbor, MI 48109, USA
}

Received 18 March 1986; accepted for publication 2 May 1986

\begin{abstract}
We present an infinite-dimensional classical integrable hamiltonian system on projective Hilbert space. We show that the equations of motion correspond to the Heisenberg ones of quantum mechanics when the hamiltonian operator is compact, and that the formulation of these equations as a classical Lax pair with parameter gives rise naturally to an infinite set of conversation laws. Further, an infinite-dimensional version of Moser's transformation for integrating classical systems is shown to relate the Heisenberg and Schrödinger pictures.
\end{abstract}

\section{Introduction}

It is well known (see refs. [1,9] for example) that the Schrödinger equation

$\mathrm{i} \dot{\psi}=H_{\mathrm{op}} \psi$

is hamiltonian on a complex Hilbert space with respect to the hamiltonian function

$H(\psi)=\frac{1}{2}\left\langle\psi, H_{\mathrm{op}} \psi\right\rangle$

and the symplectic (nondegenerate, closed) form

$w(\psi, \phi)=-\operatorname{Im}\langle\psi, \phi\rangle$

$(<\rangle$ denotes the inner product).

Motivated by the similarity of the equations of motion of nonrelativistic quantum mechanics to the Lax equations of classical integrable systems, Kupershmidt suggested recently [2] that quantum mechanical systems could be viewed as integrable systems with an infinite number of conversation. laws given by

$H_{n}(\psi)=\left\langle\psi, H_{\mathrm{op}}^{n} \psi\right\rangle$.

Now one can show also (see ref. [3] for example) that the Heisenberg equations of motion for a projection operator $P$ may be generated by the hamiltonian

(i $H_{\mathrm{op}}$, i $P$ ) where $(M, N)=\operatorname{trace}\left(M N^{*}\right)$ is an ineer product. (* denotes hermitian conjugate.) In this case the underlying symplectic manifold is $P(\mathscr{H})$, projective Hilbert space, with a quite different symplectic structure (see section 2 ). $P(\mathscr{H})$ may be regarded as an adjoint orbit of the unitary group $U(\mathscr{H})$. (Choose any skew-hermitian rank one matrix $A$ and form the orbit $U A U^{-1}, U \in U(\mathscr{H})$.)

Now using the formalism developed for integrating the equations of classical mechanics (see refs. $[4,5]$ we can write the Heisenberg equation as a Lax pair with parameter. We show that this gives rise to Kupershmidt's integrals in a natural way.

We restrict ourselves to the "classical" case $H_{\mathrm{op}}$ bounded, compact, a model which we developed rigorously in a quite different context, that of statistical analysis $[6,4]$. For the purpose of studying quantum mechanical systems the case of unbounded $H_{\text {op }}$ needs to be examined. Of course this introduces a host of difficult phenomena, but work on this case is in progress (see also ref. [7]).

What we wish to show here is that in the "classical" case an infinite-dimensional version of Moser's transformation (see refs. $[4,5]$ ) used originally to integrate classical finite-dimensional systems such as the rigid body, not only integrates our system but provides the transformation from the Heisenberg to the Schrödinger picture. 


\section{The Lax pair and conserved integrals}

We consider

$H(P)=\left(\right.$ i $\left.H_{\mathrm{op}}, \mathrm{i} P\right)$,

where $\left\langle\mathrm{i} H_{\mathrm{op}}, \mathrm{i} P\right\rangle=\operatorname{Tr}$ i $H_{\mathrm{op}}(\mathrm{i} P)^{*}=\operatorname{Tr} H_{\mathrm{op}} P . P$ is a (rank one) projection operator and $H_{\text {op }}$ is bounded, compact, hermitian. Tr denotes trace. For convenience, from now on we suppose $H_{\mathrm{op}}$ and $P$ to be skew-hermitian via the identification $P \rightarrow \mathrm{i} P, \quad H_{\mathrm{op}} \rightarrow \mathrm{i} H_{\mathrm{op}}$.

Then $H_{\mathrm{op}}$ and $P$ may be viewed as elements of the Lie algebra $u(\mathscr{H})$ of $U(\mathscr{H})$ the group of unitary operators on $\mathscr{H}$, and $H(P)$ may be regarded as the restriction of a linear function to $P(\mathscr{H})$.

$P(\mathscr{H})$ may be endowed with a symplectic form $w$ as follows: Let $X$ be a point in $P(\mathscr{H})$ (viewed as an adjoint orbit of $U(\mathscr{H})$ ). Let $\xi, \eta$ be tangent vectors to the orbit at $X$. They may be written

$\xi=\left[X, A_{1}\right], \quad \eta=\left[X, A_{2}\right], \quad A_{1}, A_{2} \in u(\mathscr{H})$.

Then $w(\xi, \eta)=\operatorname{Tr} X\left[A_{1}, A_{2}\right]$.

Thus $H(P)=\left(H_{\mathrm{op}}, P\right)$ may be viewed as a hamiltonian on $P(\mathscr{H})$ and we can show by direct calculation (see refs. [4,7]) that the hamiltonian equations of motion are

$\dot{P}=\left[P, H_{\text {op }}\right]$,

which are the Heisenberg equations of motion. (Recall all operators here are skew-hermitian.)

This is of course a classical Lax equation and is integrable from a number of points of view. (The next section gives a method for explicit integration.)

Now the Lax equation

$\dot{P}=\left[P, H_{\text {op }}\right]$

is equivalent to the Lax equation with parameter. $\left(P+\dot{\xi} H_{\mathrm{op}}\right)=\left[P+\xi H_{\mathrm{op}}, H_{\mathrm{op}}\right]$.

Consider the functions

$\operatorname{Tr}\left(P+\xi H_{\mathrm{op}}\right)^{k}, \quad k=2,3 \ldots$,

which are conserved on the flow of the Lax equa- tion with parameter. The coefficients of $\xi$ in the expansion of $\operatorname{Tr}\left(P+\xi H_{\mathrm{op}}\right)^{k}$ will be conserved on the flow of our original Lax/Heisenberg equation. Explicit calculation shows this yields the integrals

$\operatorname{Tr} H_{\mathrm{op}}^{k} P$,

which are precisely the integrals of Kupershmidt. (Further details may be found in refs. [4,7].)

\section{A transformation from the Heisenberg to the Schrödinger picture}

We have the following

Theorem. Let $P=\left(p_{i j}\right)$ be written $P=\bar{\psi}^{t} \psi$ where $\psi=\left(z_{1}, z_{2}, \ldots\right)$ is a vector of norm one in Hilbert space $\mathscr{H}$. Let $z_{i}=x_{i}+\mathrm{i} y_{i}$. Choose a basis so $H_{\mathrm{op}}$ is diagonal, $H_{\mathrm{op}}=\operatorname{diag}\left(\lambda_{1}, \lambda_{2} \ldots\right)$. Then the hamiltonian (Heisenberg) flow on $P(\mathscr{H})$ associated to $H(P)=\operatorname{Tr} H_{\mathrm{op}} P$ is generated by the hamiltonian $\bar{H}(\psi)=\frac{1}{2}\left\langle\psi, H_{\mathrm{op}} \psi\right\rangle$ on $\mathscr{H}$ with the standard Hilbert space symplectic structure, or equivalently by the hamiltonian equations of motion in Schrödinger form.

Sketch of proof: (details can be found in refs. $[4,7])$. We use a complex infinite-dimensional generalization of Moser's transformation in ref. [5]. Consider the operator

$L=P+H_{\mathrm{op}}$.

Here $P$ is a (complex) rank one perturbation of $H_{\mathrm{op}}$. The vectors $x$ and $y$ (in $z=x+\mathrm{i} y$ ) are to be viewed as vectors in real Hilbert space with inner product $\langle,\rangle_{\mathbf{R}}$. Note that $H_{\mathrm{op}}$ being compact, has discrete spectrum.

Now by the Weinstein-Aronsjan formula [8]

$$
\operatorname{det}\left((\eta-L)\left(\eta-H_{\mathrm{op}}\right)^{-1}\right)=I-\operatorname{Tr} W_{\eta}(x, y),
$$

where

$$
W_{\eta}(x, y)=\left[\begin{array}{cc}
\left\langle R_{\eta} x, x\right\rangle_{\mathbf{R}} & \left\langle R_{\eta} x, y\right\rangle_{\mathbf{R}} \\
\left\langle R_{\eta} x, y\right\rangle_{\mathbf{R}} & \left\langle R_{\eta} y, y\right\rangle_{\mathbf{R}}
\end{array}\right]
$$

and $R_{\eta}=\left(\eta I-H_{\mathrm{op}}\right)^{-1}$. 
Now one can show, for $\eta, \eta^{\prime}$ in the resolvent set of $H_{\mathrm{op}}, \operatorname{Tr} W_{\eta}$ and $\operatorname{Tr} W_{\eta^{\prime}}$, commute under Poisson bracket with respect to the standard symplectic form $-\operatorname{Im}\langle$, $>$ on Hilbert space.

Further, from

$H(x, y)=\frac{1}{4 \pi \mathrm{i}} \int_{|\eta|=R} f(\eta) \operatorname{Tr} W_{\eta}(x, y) \mathrm{d} \eta$,

where $R$ contains the spectrum of $H_{\mathrm{op}}$. With $f\left(\lambda_{j}\right)=\beta_{j}$ we find

$H=\frac{1}{2}\langle\beta x, x\rangle_{\mathbf{R}}+\frac{1}{2}\langle\beta y, y\rangle_{\mathbf{R}}, \quad \beta=\operatorname{diag}\left(\beta_{1}, \beta_{2}\right)$, setting $\beta_{j}=\lambda_{j}$,

$H=\frac{1}{2} \sum_{j} \lambda_{j}\left(x_{j}^{2}+y_{j}^{2}\right)=\frac{1}{2}\left\langle\psi, H_{\mathrm{op}} \psi\right\rangle$

(the Schrödinger hamiltonian) with hamiltonian equations

$\dot{x}=\partial H / \partial y, \quad \dot{y}=-\partial H / \partial x$,

in standard form. Further, with $P=\bar{\psi}^{t} \psi$ these equations of motion in $x$ and $y$ generate the flow
$\left(P+H_{\text {op }}\right)^{\cdot}=\left[P+H_{\text {op }}, P\right]$ or $\dot{P}=\left[H_{\text {op }}, P\right]-$ precisely the Heisenberg equations of motion. (Details of this calculation can be found in refs. [4,7].)

Thus the Schrödinger hamiltonian (6) generates the Heisenberg flow via the Moser transformation.

\section{References}

[1] P.R. Chernoff and J.E. Marsden, Lecture notes in mathematics, Vol. 425. Properties of infinite dimensional hamiltonian systems (Springer, Berlin, 1974).

[2] B.A. Kupershmidt, Phys. Lett. A 109 (1985) 136.

[3] J.E. Marsden, Canad. Math. Bull. 25 (1982) 129.

[4] A.M. Bloch, Completely integrable hamiltonian systems and total least squares estimation, Ph.D. thesis, Harvard University (1985).

[5] J. Moser, The geometry of quadrics and spectral theory, Proc. Chern Symp. 1979 (Springer, Berlin, 1980).

[6] A.M. Bloch, Bull. Am. Math. Soc. 12 (1985) 250.

[7] A.M. Bloch, A completely integrable hamiltonian system on a projective Hilbert space, to be published.

[8] T. Kato, Perturbation theory for linear operators (Springer, Berlin, 1966).

[9] R.A. Abraham and J.E. Marsden, Foundations of mechanics (Benjamin/Cummings, New York, 1978). 